\title{
IJIMI
}

International Journal of Machine Intelligence

ISSN: 0975-2927 \& E-ISSN: 0975-9166, Volume 3, Issue 5, 2011, pp-381-389

Available online at http://www.bioinfo.in/contents.php?id=31

\section{A NOVEL MORPHOLOGICAL TECHNIQUE FOR SEGMENTATION OF DOCUMENT IMAGES IN NIST DATABASE}

\author{
DHORE M.P. ${ }^{1}$, THAKARE V.M. ${ }^{2}$, KALE K.V. ${ }^{3}$ \\ 1Shivaji Science College, Congress Nagar, Nagpur, India, mpdhore@ieee.org \\ 2Department of CS \& Engg., SGB Amravati University, Amravati, India, vilthakare@yahoo.co.in \\ 32 Department of CS \& IT , Dr. B.A.M. University, Aurangabad, India, kvkale91@rediffmail.com \\ *Corresponding Author: Email- mpdhore@rediffmail.com , mpdhore@ieee.org
}

Received: September 29, 2011; Accepted: October 12, 2011

\begin{abstract}
This paper proposes a novel morphological approach for segmentation of document images in the National Institute of Standards and Technology (NIST) Database. Document Image Analysis involves transformation of any information presented on paper into an equivalent symbolic representation accessible to computer information processing. For better storage and intelligent processing, information presented on paper is now being converted into electronic form. This needs processing of documents using image analysis algorithms. The morphological approach quantitatively describes operations effective for the shape of objects in an image. The mathematical morphology describes such operations by combinations of basic set operations between an image and a small object called a structuring element. The segmentation oriented geometrical features such as size, shape, contrast, or connectivity are efficiently dealt in mathematical morphology. In this paper, morphological approach is used for segmenting the document images. The performance of the proposed method has been evaluated using many text documents. The National Institute of Standards and Technology, Technology Administration, U. S. database of document images has been used in order to carry out experimental work. The image document database used is NIST DATABASE, Federal Register Document Image Database. NIST Special Database 25 volume 1.(NISTIR 6245). The proposed technique has been tested for various documents like multi-column, multi-article, multi-font, multi-orientation and promising results are obtained. At the end the obtained performance is also compared with other well-known page segmentation algorithms.
\end{abstract}

Keywords - Document Image Analysis, Page segmentation, Document segmentation, Mathematical Morphology, Structuring Element, NIST Database.

\section{INTRODUCTION}

Document Image Analysis (DIA) [1-5] for information extraction is gaining immense importance. The information which is conventionally stored on paper is being converted into electronic form for better storage and intelligent processing. This includes information extraction [1] from document images. In order to achieve this, documents are processed using image analysis [6-9] algorithms. Document Image Analysis is a subfield of digital image processing [6-9]. This involves conversion of document images to symbolic form for modification, storage, retrieval, reuse and transmission. Document image analysis facilitates the transition from bookshelves and filing cabinets to the paperless world. Document Analysis aims at transformation of any information presented on paper and adjust to human comprehension into an equivalent symbolic representation. This symbolic representation is accessible to any kind of computer information processing.

The degree of perfection as compared with human being needs to be improved. Specialized tasks are involved in this process but technology has been able to solve this problem partially. The problem is solved to some extent with the help of high resolution scanning devices and increasing availability of computing power. This leads to accurate document analysis which facilitates high level of office automation, document analysis, document understanding [10,11], Optical Character Recognition (OCR) systems, text reading and so on.

\section{DOCUMENT IMAGE ANALYSIS}

A document is a written or printed paper that bears the original, artificial or legal form of data and can be used to furnish decisive evidence or information required. We encounter a wide variety of documents in our daily life. For e.g. the documents used to communicate information in the form of letters and newspapers, archived documents for later validation or use. The conventional documents are now being replaced by electronic documents. The challenges associated with processing of such electronic documents are considerably different from that of information extraction from digitized or converted electronic documents. 
The office automation systems are leading us to paperless offices which facilitate digital storage of documents. This helps in easy manipulation (modifications \& searches) of documents and preserving the documents for longer period. For successful implementation of office automation systems, there is an increasing demand for tools that can digitize, recognize, search and retrieve the information. The demand for tools that automatically extract, analyze and store information from physical documents for later use is increasing. These activities come under the field of Document image analysis, one of the fast growing research area. Document image analysis refers to the processing and understanding of contents of documents. Document Image Analysis means algorithms and techniques that are applied to document images to obtain a computerreadable description from pixel data[1,2].

\subsection{Text Document Analysis}

There are two main types of analysis that are applied to text in documents. One is optical character recognition (OCR) to derive the meaning of the characters and words from their bit-mapped images, and the other is pagelayout analysis to determine the formatting of the text, and from that to derive meaning associated with the positional and functional blocks (titles, subtitles, bodies of text, footnotes etc) in which the text is located. Layout analysis techniques are applied to formatted, machine-printed pages, and a type of layout analysis, forms recognition, is applied to machine-printed or handwritten text occurring within delineated blocks on a printed form. In some cases it is necessary to correct the skew of the document which is typically a result of improper paper feeding into the scanner.

The main aim of document image analysis is to recognize the text and graphics components in document images and to extract the intended information as a human would extract.

\subsection{Challenges in Document Image Processing}

A newspaper image requires several mega bytes of storage space to represent in binary form. The storage space can be drastically reduced if these document images are compressed. This allows easy manipulation of the content for many applications such as word processing and information retrieval. A document image analysis system can be employed to solve the problem of searching all relevant information about a particular individual from the huge newspaper archive. The major challenge in document image analysis is to locate text image blocks and tables, and defining appropriate algorithms for the same. Obviously this involves a number of preprocessing, segmentation and classification steps.

Beginning with the input image the document image analysis system initially tries to cancel out the noise effect and does the necessary geometric corrections. It is difficult for the computer system to identify the boundaries of an image and separate it from the textual region. Identification of table boundaries, graph boundaries and the text boundaries, and developing corresponding algorithms for table analysis, text, graphics and character recognition are some of the challenges which are to be faced in document analysis. Preprocessing consists of a series of image-to-image transformations. It helps to extract the contents of the document. The preprocessing involves compressed representation, Filtering, Binarization, Skew detection and correction.

\subsection{Page Segmentation}

Page segmentation is the process of partitioning of a page image into several rectangular regions each of which contains a unique type of data such as text-lines, line drawings, pictures, etc. A page may contain both horizontal and vertical text-lines [12,13]. Because of the allowance of such layouts, fragmentation is not tolerated due to spurious vertical cuts for horizontal textlines and the same for vertical text-lines. But in the case that several text parts are placed far apart in the same text-line, separation of these parts is tolerable. Such cases are often found in the header or footer in some page layouts. Text can have various fonts and their size may vary arbitrarily within the page. Here it is assumed that documents are scanned without severe tilt (skew), i.e., a page can be separated by right rectangle regions [14].

One of the essential issues in document analysis is the ability to handle multi-article documents containing figures and photographs. Generally a document has a visual hierarchical structure in its layout. This is called geometric structure[1] where, the hierarchy of which can be represented by a tree. Document analysis extracts this structure as a model for relationships between characters, lines, columns and a page [12-14]. A description for configuration of articles and their components, called logical structure[1] which orders the reading sequence.

\section{MORPHOLOGICAL DOCUMENT IMAGE SEGMENTATION}

The morphology approach quantitatively describe operations effective for the shape of objects in an image[13,15-17]. The mathematical morphology describes such operations by combinations of basic set operations between an image and a small object called a structuring element. It is very attractive for this purpose because it efficiently deals with geometrical features such as size, shape, contrast, or connectivity that can be considered as segmentation oriented features. One of the advantages of using morphological approach is its low computational cost. The simplification for segmentation can be efficiently achieved by filters based on opening and closing by partial reconstruction. The size of structuring element is progressively decreased to allow the introduction of more local information to improve the segmentation $[13,15-17]$. Mathematical morphology is closely related to integral geometry. It quantifies many aspects of the geometrical structure of images in a way that agrees with human intuition and perception. Mathematical morphology has been widely used for many applications of image processing and analysis [6-9]. Morphological 
processing can be employed for many purposes including pre-processing, edge detection, segmentation, and object recognition. Morphological expressions are defined as a combination of image operations, the simplest of which is the operation of erosion and dilation. The morphological approach is based on the analysis of an image in terms of some predetermined geometric shape templates known as structuring elements. The manner in which the structuring elements can be embedded into the original shape by using a specific sequence of operators leads eventually to shape classification or discrimination. The morphological operators are the filters that encode the original shape for providing features needed for shape discrimination. However the structuring elements design is difficult due to its computational intractability.

\subsection{Morphology Based Techniques}

Morphology is biological term that refers to study of form and structure[18]. Morphological operators often take a binary image and a structuring element as input and combine them using a set operator (intersection, union, inclusion, complement) $[6,8]$. They process objects in the input image based on characteristics of its shape, which are encoded in the structuring element.

Mathematical Morphology refers to a branch of nonlinear image processing and analysis that concentrates on the geometric structure within an image, it is mathematical in the sense that the analysis is based on set theory, topology, lattice, random functions, etc. $[18,19]$. As well as mathematical morphology is considered as a powerful tool to extract information from images [19]. Whereas erosion and dilation are considered the primary morphological operations and the operations of opening and closing are secondary operations and are implemented using erosion and dilation operations [6]. In this paper, Mathematical morphology is used for segmentation of text document image because morphological operations have following salient features [20]:

1. Morphological operations provide for the systematic alteration of the geometric content of an image while maintaining the stability of the important geometric characteristics.

2. There exists a well-developed morphological algebra that can be employed for representation and optimization.

3. It is possible to express digital algorithms in terms of a very small class of primitive morphological operations.

4. There exist rigorous representations theorems by means of which one can obtain the expression of morphological filters in terms of the primitive morphological operations.

In this work mathematical morphology based document image segmentation technique is proposed which provides promising results.

\subsection{Morphological Operations}

Morphology technique is used to operate on images via different operations $[6,8,13,21]$. Some of them include Erosion and Dilation, Closing and Opening, Top-hat and
Bottom-hat Transform. When a morphological operation is carried out, the origin of the structuring element is typically translated to each pixel position in the image in turn, and then the points within the translated structuring element are compared with the underlying image pixel values. The details of this comparison and the effect of the outcome depend on which morphological operator is being used. If we use both opening and closing operations in a program then structuring element or morphological mask remains same for the both operation [22].

\section{DESIGN OF PROPOSED TECHNIQUE}

In this section the design of new technique is presented and proposed as in Fig. 1, on the basis of study and analysis. It is tried to make system more automated rather than complex structure involving many subtasks. An automated system for document analysis is extremely desirable. The research work presents the development and implementation of new automation system and a new algorithmic approach for analyzing the documents.

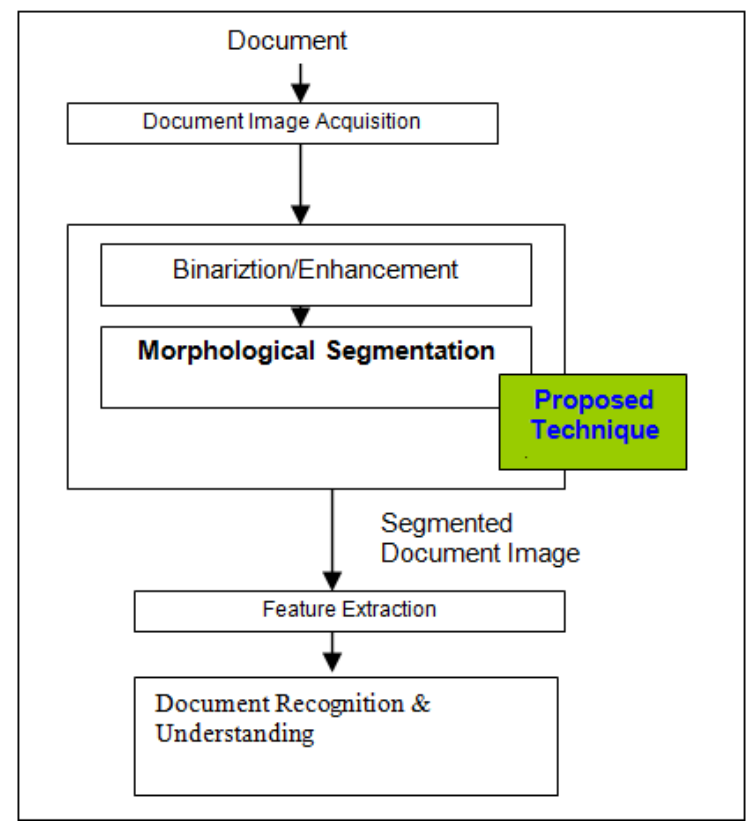

Fig. 1 Proposed Step for Document Image Analysis system

Following main criterion laid down for good segmentation technique are fully satisfied by the proposed morphological segmentation.

- It is not sensitive to the contrast in the image.

- It detects smudged and noisy regions.

- The result of segmentation is independent of whether the input image is an enhanced image or a raw image.

- It gives consistent results for a variety of images.

\section{ABOUT THE NIST DATABASE}

The performance of the proposed technique, both in terms of its effectiveness and computational efficiency 
has been evaluated using many text documents. The National Institute of Standards and Technology(NIST), Technology Administration, U. S. database of document images has been used in order to carry out experimental work. The image document database used is NIST DATABASE, Federal Register Document Image Database. NIST Special Database 25 - volume 1.(NISTIR 6245)[23]. A new, fully automated process has been developed at NIST to derive ground truth for document images. The method involves matching optical character recognition $(\mathrm{OCR})$ results from a page with typesetting files for an entire book. Public domain software used to derive the ground truth is provided in the form of Perl scripts and $C$ source codes, and includes new, more efficient string alignment technology and a word-level scoring package. With this ground-truthing technology, it is now feasible to produce much larger datasets, at much lower cost, than was ever possible with previous laborintensive, manual data collection projects. Using this method, NIST has produced a new document image database for evaluating Document Analysis and Recognition technologies and Information Retrieval systems. The database produced with this method contains scanned images, SGML-tagged ground-truth text, commercial OCR results and image quality assessment results for pages published in the 1994 Federal Register. These data files are useful in a wide variety of experiments and research. There were roughly 250 issues, comprised of nearly 69000 pages, published in the Federal Register (FR) in 1994. This volume of the database contains the pages of 20 books published in January of that year. In all there are 4711 page images provided, with 4519 of them having corresponding ground truth. This volume is distributed on two ISO-9660 CDROMs.

\subsection{Using standard NIST database}

The NIST database used for the experimentation work consists of the document images from the Federal Register(FR) 1994 of U.S. Government. The United States Government Printing Office (GPO) prints the FR each work day to record the transactions of the government. The FR is the official daily publication for Rules, Proposed Rules, and Notices of Federal agencies and organizations, as well as Executive orders and other Presidential Documents. Each issue is printed and bound into one book. In particular this database contains 20 daily FR issues for the entire month of January 1994. Each image was scanned binary at 15.75 pixels per millimeter (ppm), equaling 400 pixels per inch (ppi). The images and associated text can be used to evaluate alternative Document Analysis and Recognition (DAR) technologies, and they can be used in Information Retrieval (IR) experiments where retrieval performance can be compared between retrieving the pages of text provided with the database and retrieving the corresponding text produced by DAR. The text of each FR page in this database is formatted in Standard Generalized Markup Language (SGML). This representation is commonly used in IR experiments.

\section{RESULTS AND DISCUSSION}

The proposed morphological segmentation technique has been applied to each document image in NIST database. Some of the sample outputs obtained are presented as next. Fig. 2 shows the results after applying the morphological segmentation technique to the document image b0000003.png from the IMG-3 folder of NIST database. The proposed segmentation technique is applied to this image for various structuring elements (SE). By visually inspecting the outputs for each structuring element it is observed that promising results are obtained for SE disk and square. After comparing the results for SE disk and SE square, it is concluded that the later one is the best. The performance evaluation metrics are computed for the image. The MSE of original and segmented document image is approximately .001 and PSNR value for the same is more than $50 \mathrm{~dB}$ for square as SE. Fig. 2a. shows the original document image b0000003.png from IMG-3. Fig. 2b. and 2c. shows the intermediate results. Lastly, Fig. 2d. shows the final segmentation result.

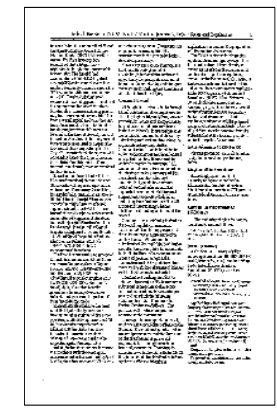

a

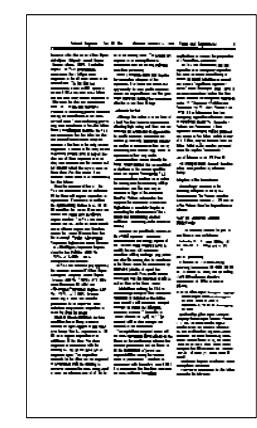

b

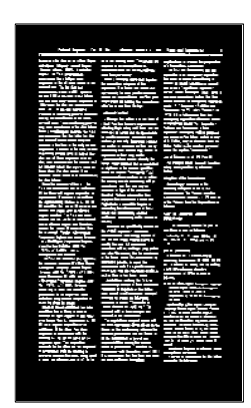

c

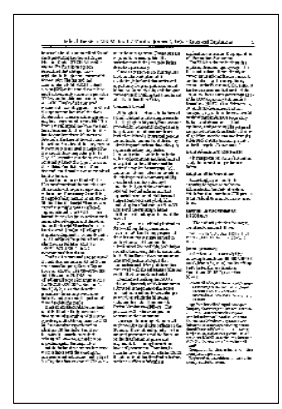

d

Fig. 2- a. Original document image b0000003.png from IMG-3, b. \& c. are intermediate results and d. The resulting segmented document image after applying proposed technique. 
6.1 Using Segment of Full Images

As the document images are scanned versions of A4 size papers, they are of very big sizes. The size of the NIST document images vary between $3000 \times 3000$ pixels to $4000 \times 4000$ pixels. It is very difficult to compare the outputs with naked eye. The distinction between the original and segmented document image is much more evident if we consider a small portion of the whole document image. This is shown in Fig. 3. As the contents of the portion of document image are visible, one can very easily observe the intermediate results as depicted in Fig. 3b. and Fig. 3c. The final morphologically segmented image is shown in Fig. $3 \mathrm{~d}$. Here it is observed that segmented image is nearly same as that of original image shown in Fig. 3a.

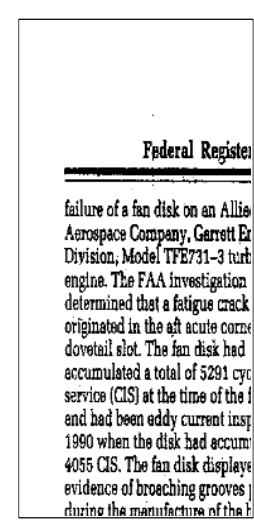

a

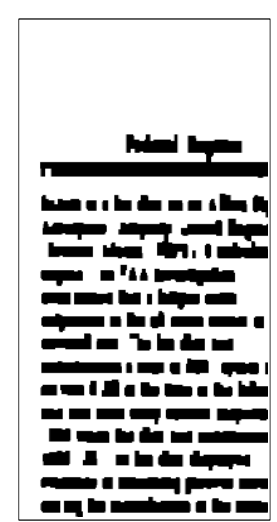

b

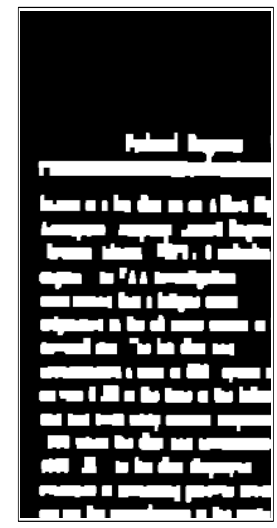

C

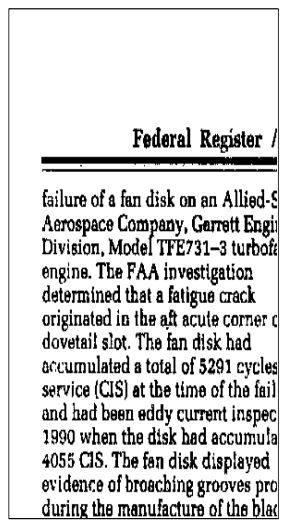

d

Fig. 3- a. Small portion of original document image b0000003.png from IMG-3, b. \& c. are intermediate results and d. small portion of the resulting segmented document image after applying proposed technique.

\subsection{Using Multi-font and Multi-directional Document Images}

Fig. 4 shows the results after applying the morphological segmentation technique to the document image b0000109.png from the IMG-5 folder of NIST database. This particular document image consists of text in various fonts and font sizes. The document image also contains the orientation of text in two directions, vertical as well as horizontal. The proposed segmentation technique is applied to this image for various structuring elements (SE). By visually inspecting the outputs for each structuring element it is observed that promising results are obtained for SE disk and square. After comparing the results for SE disk and SE square, it is concluded that the later one is the best. The performance evaluation metrics are computed for the image. Fig. $4 a$. shows the original document image b0000109.png from IMG-5. Fig. 4b. shows the final segmentation result.

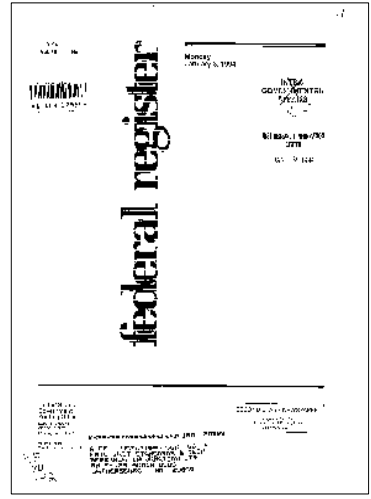

a.

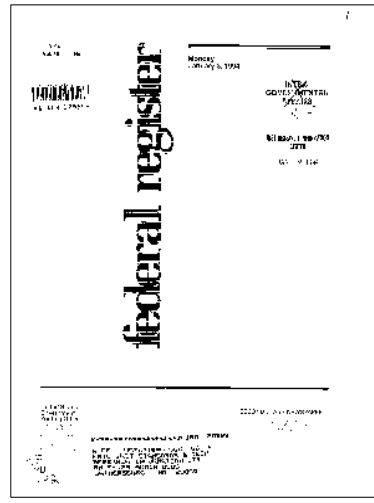

b.

Fig. 4- a. Original document image b0000109.png from IMG-5 and b. The resulting segmented document image after applying proposed technique.

\subsection{Using Mixed mode Document Images}

Fig. 5 shows the results after applying the morphological segmentation technique to a segment of document image b0000109.png from the IMG-5 folder of NIST database. This particular document image consists of mixed mode text. It contains printed as well as handwritten text. 
Moreover the text consists of varying font sizes. The proposed segmentation technique is applied to this image for various structuring elements (SE). By visually inspecting the outputs for each structuring element it is observed that promising results are obtained for SE disk and square. After comparing the results for SE disk and SE square, it is concluded that the results obtained for SE square are the best. The performance evaluation metrics are computed for the image. Fig. $5 \mathrm{a}$. shows the segment of original document image b0000109.png from IMG-5. Fig. 5b. shows the final segmentation result.

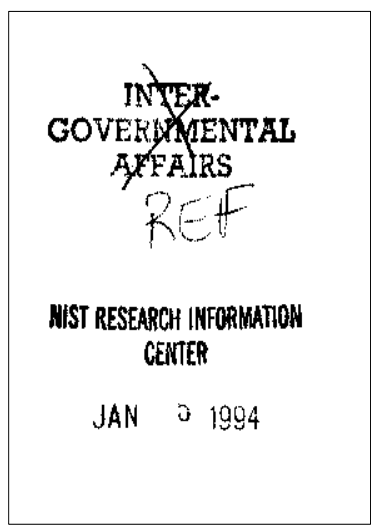

a

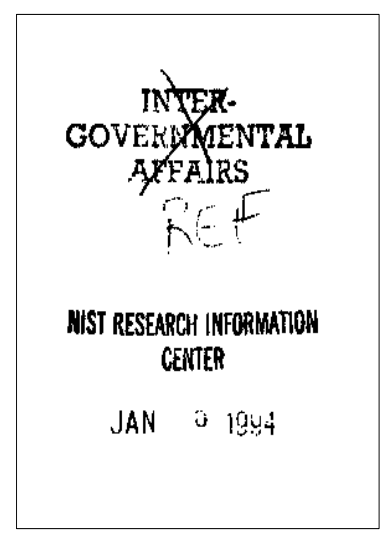

b

Fig. 5- a. A segment of original document image b0000109.png from IMG-5 and b. The resulting segmented document image after applying proposed technique.

\subsection{Using Graphics Document Images}

Fig. 6 shows the results after applying the morphological segmentation technique to document image b0000030.png from the IMG-3 folder of NIST database. This particular document image consists of graphics data. It contains information in the form of a geographical map. The proposed segmentation technique is applied to this image for various structuring elements (SE). By visually inspecting the outputs for each structuring element it is observed that promising results are obtained for SE disk and square. After comparing the results for SE disk and SE square, it is concluded that the results obtained for SE square are the best. The performance evaluation metrics are computed for the document image. Fig. $6 \mathrm{a}$. shows the original document image b0000030.png from IMG-3. Fig. 6b. shows the final segmentation result.

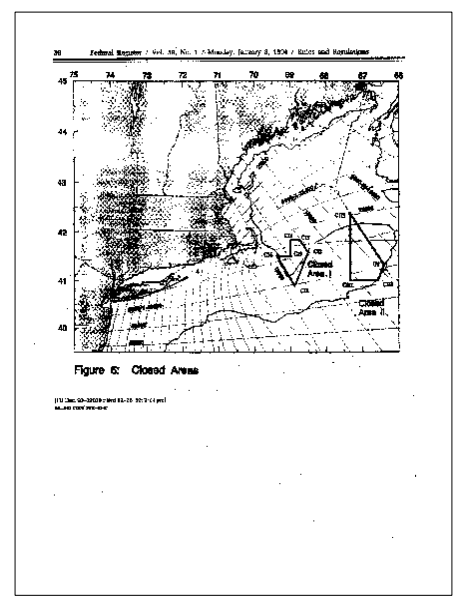

a.

Fig. 6- a. Original document image b0000030.png from IMG-3 and b. The resulting segmented document image after applying proposed technique.

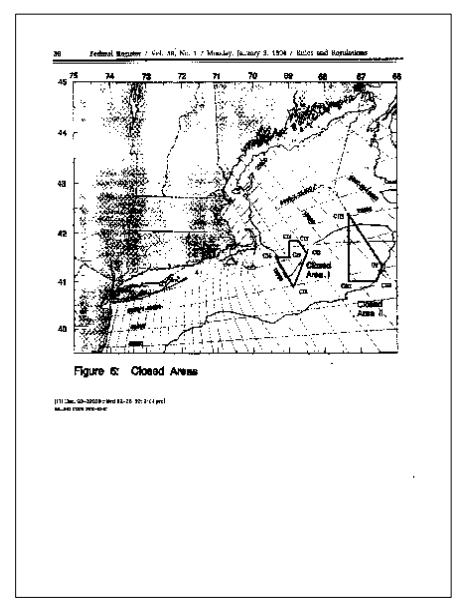

b. 
6.5 Using Segment of Graphics Document Images

Fig. 7 shows the results after applying the morphological segmentation technique to document image b0000030.png from the IMG-3 folder of NIST database. This particular document image is a segment of graphics data. It contains information in the form of a geographical map. The proposed segmentation technique is applied to this image for various structuring elements (SE). By visually inspecting the outputs for each structuring

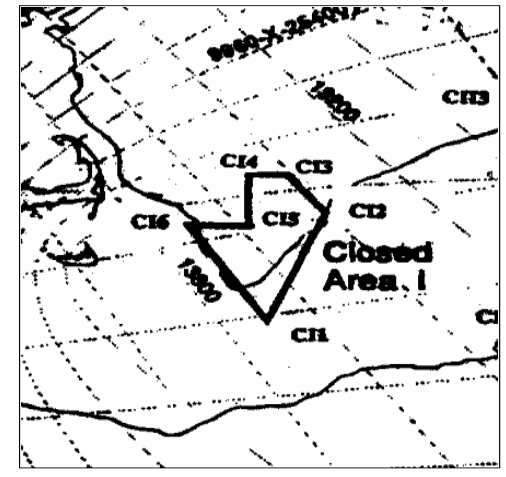

a. element it is observed that promising results are obtained for SE disk and square. After comparing the results for SE disk and SE square, it is concluded that the results obtained for SE square are the best. The performance evaluation metrics are computed for the document image. Fig. 7a. shows the segment of original document image b0000030.png from IMG-3. Fig. 7b. shows the final segmentation result.

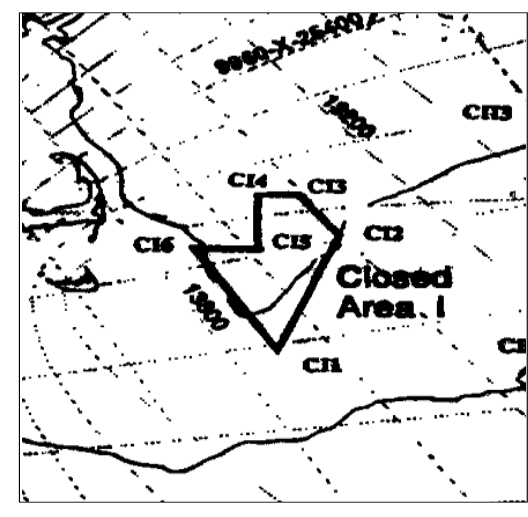

b.

Fig. 7- a. Segment of original document image b0000030.png from IMG-3 and b. The resulting segmented document image after applying proposed technique.

\section{PERFORMANCE EVALUATION}

As mentioned earlier, the database used for the experimentation purpose is the NIST Special Database 25 - volume 1 (NISTIR 6245). In particular this database contains 20 daily FR issues for the entire month of January 1994. In the database, the scanned copies of the pages of FR books of 20 working days in January 1994 is organized into 20 different folders such as IMG-3, IMG-4, IMG-5, .... IMG-31. The numerals in the folder name represent the date in the month, January 1994. In table 1 we give the details of document images in the database. The values of performance evaluation measures such as mean, standard deviation, MSE and PSNR are calculated for each document image in every folder of the above mentioned document image database. The experimentation work has been done on NIST Document image database [23] for various structuring elements with varying parameter values.

Table 1- Details of document images in the NIST database

\begin{tabular}{|c|c|c|c|}
\hline Sr.No. & Folder name & File names & Total no. of files \\
\hline 1 & IMG-3 & B0000001.PNG to B0000240.PNG & 240 \\
\hline 2 & IMG-4 & B0000241.PNG to B0000498.PNG & 254 \\
\hline 3 & IMG-5 & B0000499.PNG to B0000651.PNG & 151 \\
\hline 4 & IMG-6 & B0000653.PNG to B0000945.PNG & 288 \\
\hline 5 & IMG-7 & B0000947.PNG to B0001261.PNG & 313 \\
\hline 6 & IMG-10 & B0001263.PNG to B0001445.PNG & 183 \\
\hline 7 & IMG-11 & B0001447.PNG to B0001616.PNG & 170 \\
\hline 8 & IMG-12 & B0001617.PNG to B0001888.PNG & 268 \\
\hline 9 & IMG-13 & B0001889.PNG to B0002280.PNG & 390 \\
\hline 10 & IMG-14 & B0002281.PNG to B0002518.PNG & 234 \\
\hline 11 & IMG-18 & B0002519.PNG to B0002724.PNG & 204 \\
\hline 12 & IMG-19 & B0002725.PNG to B0002924.PNG & 200 \\
\hline 13 & IMG-20 & B0002925.PNG to B0003311.PNG & 380 \\
\hline 14 & IMG-21 & B0003313.PNG to B0003512.PNG & 198 \\
\hline 15 & IMG-24 & B0003513.PNG to B0003632.PNG & 118 \\
\hline 16 & IMG-25 & B0003633.PNG to B0003650.PNG & 18 \\
\hline 17 & IMG-26 & B0003651.PNG to B0003769.PNG & 118 \\
\hline 18 & IMG-27 & B0003771.PNG to B0003980.PNG & 207 \\
\hline 19 & IMG-28 & B0003981.PNG to B0004232.PNG & 250 \\
\hline 20 & IMG-31 & B0004233.PNG to B0004545.PNG & 307 \\
\hline
\end{tabular}

Note: Some file numbers are missing in some folders. 
It is observed that appreciating segmentation results are obtained for Structuring element, SE = (disk, 3) and SE = (square, 4). After analyzing the data values of the image quality measures, it is seen that the mean square error(MSE) in case of SE = (square, 4$)$ is substantially less than the mean square error in case of $\mathrm{SE}=($ disk, 3 ). A lesser value of mean square error results in high values of peak to signal noise ratio(PSNR). For a sample of nearly 4519 document images from NIST database, for $\mathrm{SE}=($ square, 4$)$ the error is less than 0.01 for 4431 documents. Thus the success rate is $98 \%$.

The obtained performance is much better as compared with other well-known page segmentation algorithms [24]. The table 2 shows the comparison of performance of these well-known techniques with the one proposed in this paper.

Table 2- Comparison of performance of proposed technique with known techniques.

\begin{tabular}{|c|l|l|l|}
\hline Sr. No. & Technique/Algorithm & Data set & Success rate in \%. \\
\hline 1 & Voronoi[24] & $\begin{array}{l}\text { University of Washington III data set. } \\
978 \text { document images. }\end{array}$ & 94.60 \\
\hline 2 & Docstrum[24] & $\begin{array}{l}\text { University of Washington III data set. } \\
978 \text { document images. }\end{array}$ & 94.09 \\
\hline 3 & XY-cut[24] & $\begin{array}{l}\text { University of Washington III data set. } \\
978 \text { document images. }\end{array}$ & 82.92 \\
\hline 4 & Proposed technique & $\begin{array}{l}\text { NIST Database. } \\
\text { 4519 document images. }\end{array}$ & $\mathbf{9 8}$ \\
\hline
\end{tabular}

\section{REFERENCES}

[1] George Nagy (2000) IEEE transactions on PAMI, Vol. 22 No. 1, pp. 38-62.

[2] Rangachar Kasturi, Lawrence O'Gorman and Venu Govindaraju (2002) Sadhana, Volume 27, Number $1 /$ pp. 3-22.

[3] Wong K.Y., Casey R.G., Wahl F.M. (1982) IBM Journal of Research and Development 26,6, pp.647-656.

[4] Jurgen Schurmann, Norbert Bartneck, Thomas Bayer, Jurgen Franke, Eberhard Mandler, and Matthias Oberlander (1992) Proceedings of the IEEE, Vol. 80, No. 7, pp.1101-1119.

[5] Nagy G., Seth S. and Viswanathan M. (1992) Computer, vol. 25, pp. 10-22.

[6] Rafael C. Gonzalez, Richard E. Woods, "Digital image processing" (2nd Edition) Pearson Education.

[7] Rafael C. Gonzalez, Richard E. Woods, Steven L. Eddins, "Digital image processing using Matlab".

[8] Milan Sonka, Vaclav Hlavac, Roger Boyle, "Image Processing, Analysis and Machine Vision" (2nd Edition).

[9] Chanda B. and Dutta Majumdar D. (2007) Digital Image Processing and Analysis, PHI.

[10] Cattoni R., Coianiz T., Messelodi S., Modena C. M. (1998) ITC-IRST, Italy Technical Report.

[11] Kanai J., Nartker T.A., Rice S.V. and Nagy G. (1993) Proceedings of the $2^{\text {nd }}$ International Conference on Document Analysis and Recognition, pp. 424 - 427, Tsukuba, Japan, IEEE Computer Society.

[12] Dhore M.P., Thakare V.M., Kale K.V. (2009) Proceedings of National Seminar on
Information Technology Enabled Services (I TeS-2009).

[13] Dhore M.P., Thakare V.M., Kale K.V. (2009) Proceedings of National Conference on Advanced Technologies in Computing \& Networking (ATCON - 2009).

[14] Dhore M.P., Thakare V.M., Kale K.V. (2008) Proceedings of National Conference on Emerging Trends in Communication \& Information Technology (ETCIT-2008).

[15] Mandal S., Choudhari S. P., Das A. K., Bhabatosh Chanda(2006) Proceedings of the Second International Conference on Document Image Analysis for Libraries(DIAL'06.

[16] Su Chen, Robert M. Haralick and Ihsin T. Phillips(1995) Proceedings of IEEE, pp. 761-764.

[17] Belaïd Y. and Belaïd A. (2004) Proceedings of the $17^{\text {th }}$ International Conference on Pattern Recognition (ICPR'04) IEEE.

[18] Edward R. Dougherty, Roberto A. Lotufo, "Hands-on Morphological Image Processing", SPIE Press.

[19] Serra J. (1989) Image Analysis and Mathematical Morphology: Theoretical Advances, Academic Press. London.

[20] Charles R. Giardina and Edward R. Dougherty (1998) Morphological Methods in Image and Signal Processing, Prentice-Hall, Inc.

[21] Dhore M.P., Thakare V.M., Kale K.V. (2010) Proceedings of International Conference on Emerging Trends in Computer Science, Communication and Information Technology (CSCIT2010). 
[22] Philippe Salembier and Montse Pardas (1994) IEEE Transactions on Image Processing, Vol. 3. No. 5 .

[23] Manual of NIST DATABASE, Federal Register Document Image Database. NIST Special Database 25 - volume 1.(NISTIR 6245).

[24] Song Mao, Tapas Kanungo (2001) IEEE Transactions on Pattern Analysis and Machine Intelligence vol. 23, no. 3, pp. 242-256. 